\title{
Hyperbrain network properties of guitarists playing in quartet
}

\author{
Viktor Müller, ${ }^{1}$ Johanna Sänger, ${ }^{1}$ and UIman Lindenberger ${ }^{1,2,3}$ \\ ${ }^{1}$ Center for Lifespan Psychology, Max Planck Institute for Human Development, Berlin, Germany. ${ }^{2}$ European University \\ Institute, San Domenico di Fiesole (FI), Italy. ${ }^{3}$ Max Planck UCL Centre for Computational Psychiatry and Ageing Research, \\ London, England, and Berlin, Germany \\ Address for correspondence: Dr Viktor Müller, Center for Lifespan Psychology, Max Planck Institute for Human Development, \\ Lentzeallee 94, 14195 Berlin, Germany.vmueller@mpib-berlin.mpg.de
}

\begin{abstract}
When playing music in an ensemble, musicians need to precisely coordinate their actions with one another. As shown in our previous studies on guitar duets, interbrain synchronization plays an essential role during such interactions. In this study, we simultaneously recorded electroencephalograms from four guitarists during quartet playing, to explore the extent and the functional significance of synchronized cortical activity across four brains. We found that hyperbrain networks based on intra- and interbrain connectivity across four brains dwell on higher frequencies for intrabrain communication and on lower frequencies for interbrain connections. The hyperbrain networks show small-world topology, with a tendency to become more random at lower frequencies and more regular at higher frequencies, such that local efficiency increases and global efficiency decreases with higher frequencies. We identified two different types of information flow within the hyperbrain networks-intra- versus intermodular-which are based on hyperbrain modules that include nodes from two, three, or even four brains. Furthermore, we found that hyperbrain networks are unstable and change their structure over time, often as a function of musical context. Our findings demonstrate complex hyperbrain network interactions in a guitar quartet and point to mechanisms that support temporally coordinated joint action.
\end{abstract}

Keywords: intra- and interbrain coupling; graph-theoretical approach; EEG hyperscanning; phase synchronization; hyperbrain networks; social interaction

\section{Introduction}

In daily life, people must often coordinate their actions with those of others or modify their own actions in response to the continuously changing actions of a partner. ${ }^{1,2}$ Recent research indicates that synchronized brain activity, especially interbrain synchronization, accompanies coordinated behavior and plays a crucial role in social interaction. ${ }^{1,3,4}$ Oscillatory couplings have also been observed for other biological functions, such as respiration and cardiac activity during choir singing. ${ }^{5}$ However, the neural mechanisms that implement interpersonally coordinated behavior and support social interaction remain elusive, ${ }^{2,6,7}$ especially when interaction involves a group of more than two people.

In several studies with guitarist duets, ${ }^{8-11}$ we found that synchronization within and between the brains is an inevitable feature of interpersonal action coordination (IAC). Furthermore, these synchronization patterns build up so-called hyperbrain networks bringing together two (or even more) brains. It has also been found that, within this (hyperbrain) network, intrabrain connections primarily involve higher frequencies (e.g., beta), whereas interbrain connections primarily operate at lower frequencies (e.g., delta and theta). ${ }^{9}$ As shown in previous studies, synchronization patterns and hyperbrain network properties depend on musical situation and musical roles (e.g., leader versus follower) of guitarists. ${ }^{9-11}$ Moreover, we identified modules composed of nodes from two brains, so-called hyperbrain modules. ${ }^{9,10}$ In accordance with graph-theoretical approaches and definitions of community structures or modules, the information 
flow within modules tends to be higher than the flow between modules. ${ }^{12-14}$ Thus, nodes belonging to hyperbrain modules and areas captured by these nodes may point to brain regions that are strongly interconnected and implement, therefore, mechanisms of IAC (e.g., increase in interbrain connectivity in situations with high demands on musical coordination). ${ }^{9,10}$

The aim of the study was to describe hyperbrain networks and synchronization patterns of guitarists playing guitar in quartet. Synchronization patterns were quantified using electroencephalography (EEG) hyperscanning (simultaneous EEG recording from multiple brains; four in this case) and following time-frequency decomposition. EEG connectivity matrices were determined using phase synchronization algorithms at the surface electrode level. Networks binding four brains together are complex networks with corresponding intrinsic and extrinsic dynamics that have never been described before. There are studies with simultaneous recording of four people, but they have not been focused on the hyperbrain network as a whole. ${ }^{15,16}$ Very recently, Dikker et al. ${ }^{17}$ investigated brain-to-brain synchrony in the classroom and showed that synchronous EEG activity across students predicts student class engagement and social dynamics. Chang et al. ${ }^{18}$ also reported body-sway synchrony (recorded with motion capture) in string quartets, with a greater influence of the leader on the others. Critically, we focused on hyperbrain network properties and hyperbrain network architecture based on community structures and their changes across frequency and time. Our analyses were carried out on musical segments indicating different situations of interaction and communication between the quartet members. The segments were chosen by a professional musician using video and audio recordings. We expected that hyperbrain modules composed of nodes or electrodes from two, three, or even four brains that facilitate information flow within the shared module are important mechanisms of IAC. We also analyzed hyperbrain dynamics (i.e., temporal changes in network properties and modular organization). It was expected that synchronization patterns and corresponding network architecture gathered at fine-tuned time scales would provide richer information than corresponding connectivity data gathered during the entire sequence period. In particular, we expected that the number of mod- ules and, correspondingly, the number of hub and connector nodes would increase or decrease dependent on musical situation (e.g., situations with high demands on musical coordination might lead to a decrease in the number of modules and corresponding increase of intramodular flow with a strong interbrain connectivity, while situations with more differential roles across guitarists might increase the number of modules and correspondingly strengthen the intra- and intermodular connections).

\section{Methods}

\section{Participants}

A quartet of professional guitarists (Cuarteto Apasionado, Berlin) participated in the study. Participants' mean age was 46.5 years $(\mathrm{SD}=1.7)$. All participants (females) were right-handed and had been playing the guitar professionally for more than 35 years $($ mean $=37.8$ years, $S D=1.3$ ). The ethics committee of the Max Planck Institute for Human Development approved the study, and it was performed in accordance with the ethical standards established in the 1964 Declaration of Helsinki. All participants volunteered for this experiment and gave their written informed consent before their inclusion in the study.

\section{EEG data acquisition and preprocessing}

EEG measurement took place while the quartet played two music pieces: Libertango (Astor Piazolla) and Comme un Tango (Patrick Roux). These musical pieces were chosen with regard to different aspects of IAC, such as different phases of the musical piece, periods of harmonic consonance as judged by the expert musician changes of tempo, and phases of different musical complexity. The guitarists sat in a semicircle or formed a light bow. EEG was simultaneously recorded using four electrode caps with $28 \mathrm{Ag} / \mathrm{AgCl}$ EEG active electrodes each, placed according to the international 10-10 system, with the reference electrode at the right mastoid and the ground electrode at the AFz position. Vertical and horizontal electrooculograms were recorded to control for eye blinks and eye movements. The sampling rate was $5000 \mathrm{~Hz}$. Recorded frequency bands ranged from 0.01 to $1000 \mathrm{~Hz}$. All amplifiers (BrainAmps MR and BrainAmps ExG, Brain Products, Gilching, Germany) were connected to the same computer through PCI interfaces and synchronized using BrainVision recorder software. Through 
one microphone each, the sounds of the guitars were recorded on four ExG channels, simultaneously with the EEG recordings. In addition, video and sound were recorded using a video camera connected to the EEG computer through a FireWire socket and Video Recorder software as a component of the BrainVision software (Brain Products), synchronized in this way with EEG data acquisition. Data were rereferenced offline to an average of the left and right mastoid separately for each participant. Eye movement correction was accomplished by independent component analysis. ${ }^{19}$ Thereafter, artifacts from head and body movements were rejected by visual inspection. Spontaneous EEG activity was resampled at $1000 \mathrm{~Hz}$ and divided into 5-s epochs. Event markers were set by a professional musician and correspond to different musical situations. The list of the events and their short description for both music pieces is presented in Table S1 (online only). There were 10 and 14 segments in Libertango and Comme un Tango, respectively, that were free of artifacts for all four guitarists. To determine directed couplings we used the adaptive integrative coupling index (aICI). In contrast to our earlier studies, ${ }^{5,9,11}$ we used an adaptive algorithm that allowed us to calculate this coupling index depending on the angle of phase differences determined in a given time window. ${ }^{20}$ Coupling (aICI) was determined for nine different frequencies of interest (FOIs): 2.5, 5, 10, 15, 20, 25, 30,40 , and $60 \mathrm{~Hz}$. This coupling measure was then used to construct hyperbrain networks. To investigate the network topology, four different network characteristics (clustering coefficient (CC), characteristic path length $(\mathrm{CPL})$, local efficiency $\left(E_{\text {local }}\right)$, and global efficiency $\left.\left(E_{\text {global }}\right)\right)$ and two small-world (SW) coefficients $(\sigma$ and $\omega$ ) were determined (see Supplementary Materials, online only, for details).

\section{Results}

\section{Phase synchronization and brain connectivity patterns}

In Figure 1, we illustrate the time course of phase synchronization patterns for each of the four guitarists $(\mathrm{A}, \mathrm{B}, \mathrm{C}$, and $\mathrm{D})$ between the Fz electrode and all other electrodes within and between the brains at the FOI $(2.5 \mathrm{~Hz})$. Corresponding recordings of acoustic channels for each of the guitarists are presented below the respective synchronization pattern. Although the four guitarists showed different synchronization patterns, the coupling (red for positive and blue for negative phase difference; see Supplementary Materials, online only, for details) and decoupling (depicted in green) phases possess specific rhythmicity, which is mostly related to the notes played.

The brain maps for directed coupling (left panels) across the entire time sequence of $5 \mathrm{~s}$ and coupling strength distribution (right panels) within and between the brains are presented in Figure $1 \mathrm{~B}$ and $\mathrm{C}$, respectively. For better visualization, the strongest within- and between-brain connections are depicted.

\section{Network metrics as a function of costs}

In Figure 2A, we present various network metrics (CC, CPL, $E_{\text {local }}$, and $\left.E_{\text {global }}\right)$ and SW coefficients ( $\sigma$ and $\omega$ ) as functions of costs, defined as ratio of the number of actual connections divided by the maximum possible number of connections in the network, among real, regular (lattice), and random networks for an FOI of $2.5 \mathrm{~Hz}$. CC and $E_{\text {local }}$ at this FOI increase continuously with higher costs in random and real networks but remain stable in the range $7-50 \%$ of costs in regular networks after a strong increase at the beginning. CPL decreases in real and random networks and is absent in regular networks because of infinite CPL in remote neighbors; this is also the reason for absence of CPL in real networks at the costs under $17 \%$ and in the random networks at the costs under $9 \%$. In contrast, $E_{\text {global }}$ (inverse shortest path length) is given in all three networks at all costs and increases with higher costs, correspondingly. Real networks possess much higher global efficiency than regular networks but lower global efficiency than random networks. The SW coefficient $\sigma$ is absent at the costs under 9\% (because of absence of CPL) and decreases slightly with higher costs after a sharp increase at the beginning. In nearly all cases (exception is $9 \%$ of costs), this SW coefficient is always higher than 1 , indicating that the hyperbrain quartet network is a small-world network (SWN) at all costs, at least until the $50 \%$ cost presented here. The other SW coefficient $\omega$ lies after the costs of $10 \%$ in the positive range, indicating an SWN with more random characteristics. Taking into account all the information (as well as data from other frequencies not presented here), we decided to further describe the network characteristics at the cost level of $25 \%$, which provides stable behavior of the investigated networks. 


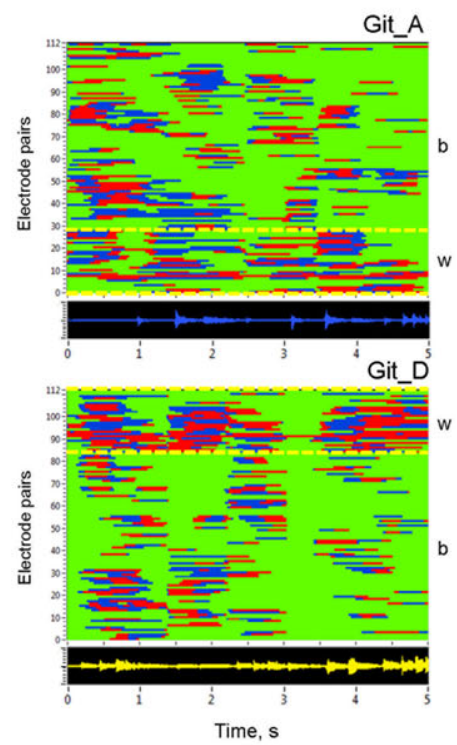

B Within-brain connectivity

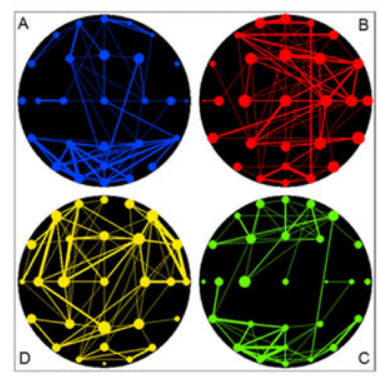

C

Between-brain connectivity

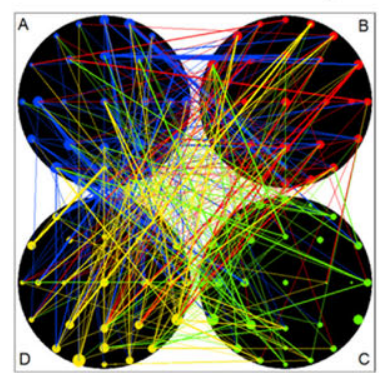

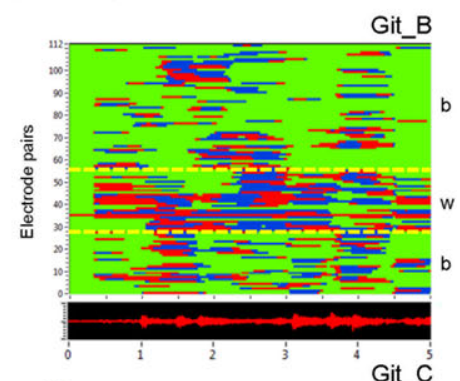

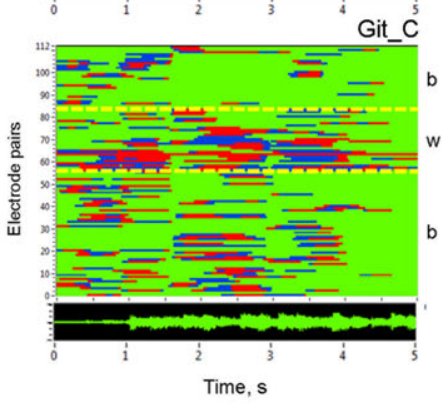

Within-brain strength distribution

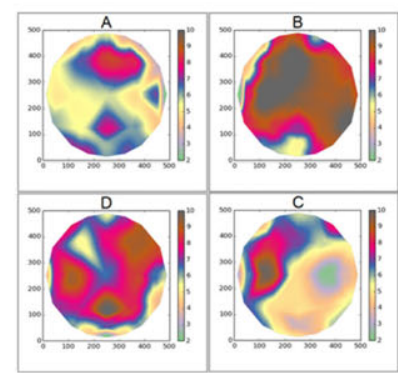

Between-brain strength distribution

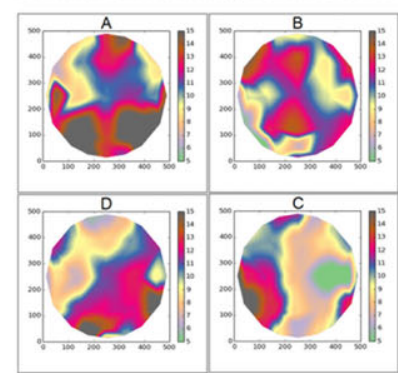

Figure 1. Phase synchronization patterns at the FOI $(2.5 \mathrm{~Hz})$ and corresponding hyperbrain networks for four guitarists. (A) Phase synchronization patterns for guitarists $\mathrm{A}, \mathrm{B}, \mathrm{C}$, and D display connectivity between $\mathrm{Fz}$ and all other electrodes within and between the brains ( $28 \times 4=112$ lines). Coding of the phase difference: negative range $=$ blue; positive range $=$ red; and out of range (nonsynchronization) = green. Dotted yellow horizontal lines indicate the boundaries for the within- and between-brain coupling (marked with $w$ and b, respectively). Representations of audio tracks of each of the guitarists are presented below the corresponding synchronization pattern. (B) Brain connectivity maps and topological distribution of strength within the brain. (C) Brain connectivity maps and topological distribution of strength for between-brain connections. The connections represent the aICI values above the threshold (within-brain connectivity: aICI $>0.50$; between-brain connectivity: aICI $>0.45$ ). The size of the circle in the brain connectivity maps represents the strength of the nodes (electrodes), and color codes represent the corresponding guitarist. In the strength distribution maps, high intensity of out-strength (accumulation of the big circles) is depicted by dark red or brown. 
A

Network metrics as a function of costs $(\mathrm{FOI}=2.5 \mathrm{~Hz})$
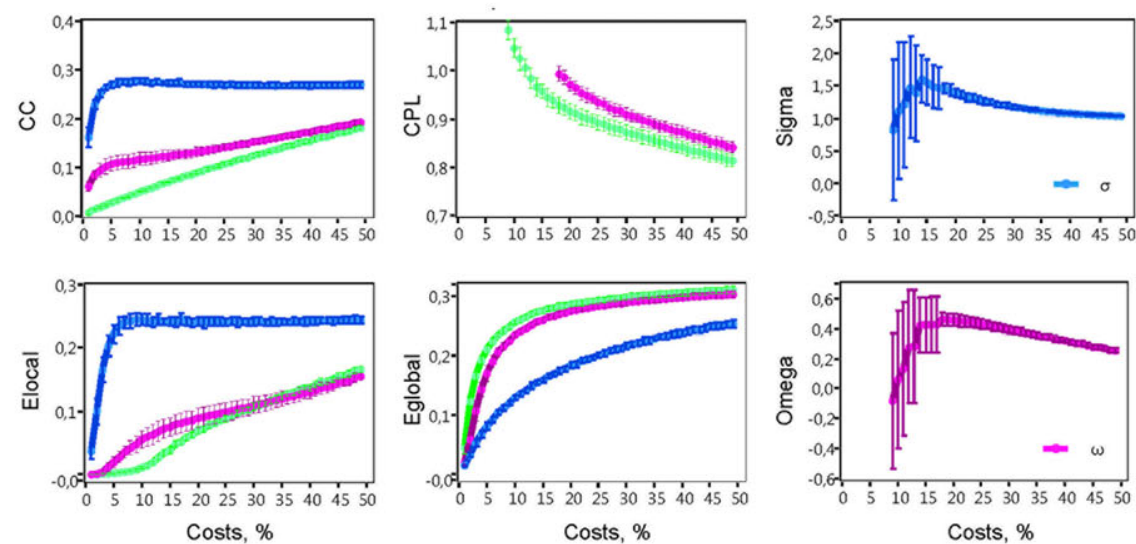

B

Network metrics as a function of frequency (Costs $=25 \%$ )
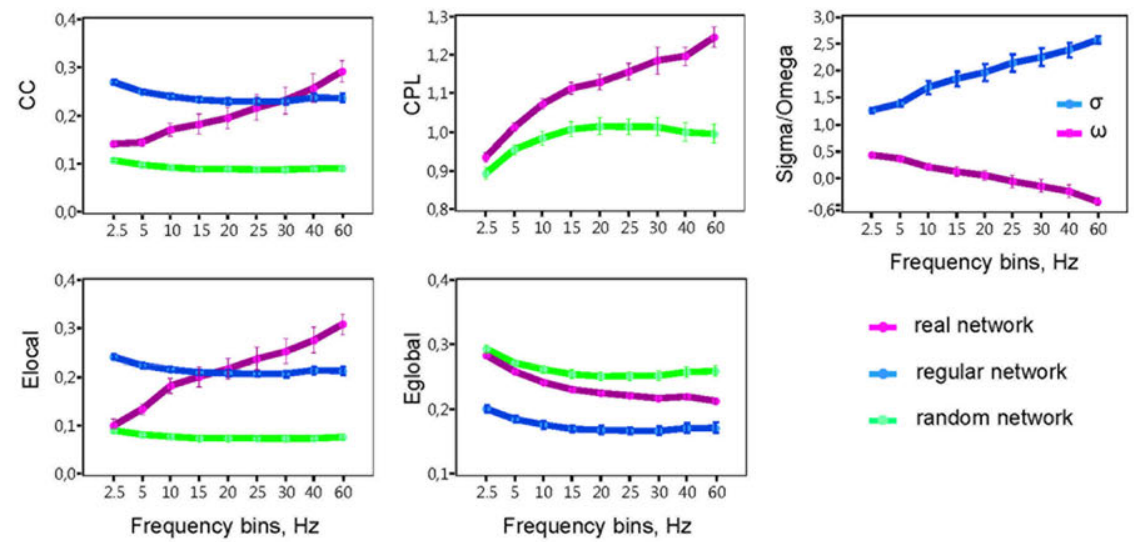

$\sim$ real network

$\approx$ regular network

-.- random network

C

Network strengths as a function of frequency (Costs $=25 \%$ )

$\mathrm{Fz}$

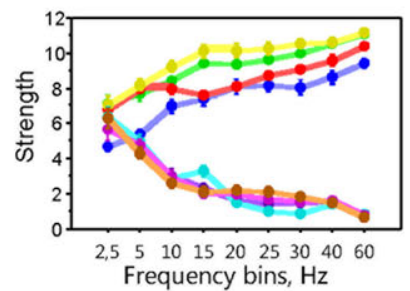

$\mathrm{Cz}$

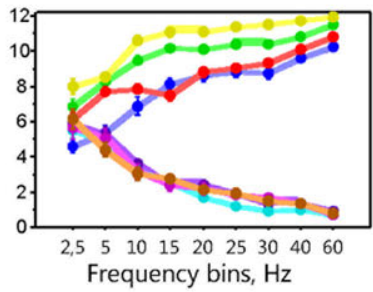

$\mathrm{Pz}$

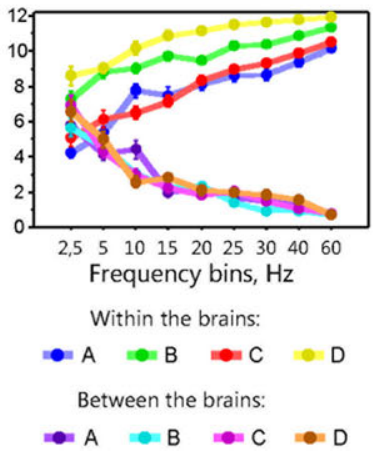

Figure 2. Hyperbrain network metrics as a function of wiring costs and frequency in the real, regular, and random networks. (A) Hyperbrain network metrics as a function of costs (FOI $=2.5 \mathrm{~Hz})$. (B) Hyperbrain network metrics as a function of frequency (costs $=25 \%$ ). (C) Network strengths as a function of frequency (costs = 25\%) in four guitarists for intra- and interbrain connections. Hyperbrain network properties averaged across all musical sequences are presented: clustering coefficient (CC), characteristic path length $(\mathrm{CPL})$, local efficiency $\left(E_{\text {local }}\right)$, global efficiency $\left(E_{\text {global }}\right)$, and small-world coefficients $(\boldsymbol{\sigma}$ and $\omega)$. The CPL of regular networks always equaled infinity and is, therefore, not presented in the diagram. This is also why some CPL values in real and random networks as well as some small-world coefficients ( $\boldsymbol{\sigma}$ and $\boldsymbol{\omega})$ are missing. 
Moreover, this cost level led to a stronger threshold than that determined by the surrogate data procedure. In other words, networks at this cost or sparsity level included highly significant connections only.

\section{Network metrics as a function of frequencies}

Figure 2B displays hyperbrain network characteristics as a function of frequency for $25 \%$ of costs. It can be seen that CC and, correspondingly, $E_{\text {local }}$ increase with higher frequency, indicating an increase of segregation processes in the hyperbrain network. CPL increases with higher frequency, and $E_{\text {global }}$ decreases correspondingly. Both indicate a decrease of integration processes with higher oscillation frequency in hyperbrain networks. The SW coefficient $\sigma$ increases with higher frequency and is always higher than 1 , indicating that the investigated hyperbrain network is an SWN at all frequencies, at least at the chosen cost level. The second SW coefficient $\omega$ decreases continuously with higher frequency and lies in the positive range at low frequencies (2.5$15 \mathrm{~Hz}$ ) and in the negative range at high frequencies $(20-60 \mathrm{~Hz})$, indicating an SWN with more random characteristics at low frequencies and with more regular characteristics at high frequencies.

Intra- and interbrain strengths at different frequency bins averaged across different musical sequences are displayed in Figure $2 \mathrm{C}$ for the three midline electrodes ( $\mathrm{Fz}, \mathrm{Cz}$, and $\mathrm{Pz}$ ), and for the four guitarists separately. It can be seen that intra- and interbrain strengths vary in different guitarists as a function of frequency. Overall, intrabrain strengths increase and interbrain strengths decrease with higher frequency, as indicated by a significant coupling (within versus between the brains) $\times$ frequency interaction (see Table S3, online only, for details).

\section{Modularity and overlapping architecture of hyperbrain networks}

As shown in Figure S1A (online only) for networks with oscillation frequency of $2.5 \mathrm{~Hz}$, modularity (M) decreases with higher costs in all the three network types, whereby the M-values for real networks always lie between the values for random and regular networks. In line with this, the number of modules also decreases and comprises about 4-5 modules for all the three network types for costs between $12 \%$ and $30 \%$. For networks with costs of interest $(25 \%)$, modularity increases with higher frequency, and the number of modules only slightly increases or remains mostly the same (Fig. S1B, online only). As described in the Supplementary Materials (online only), we compared the modularity of each hyperbrain network to the modularity distribution of random networks $(N=100)$ (i.e., networks with the same number of nodes and edges as the original network). ${ }^{21}$ The modularity of all the networks was always significantly higher than the modularity of its random counterpart (see Table S2, online only, for detail). Figure S1C (online only) displays the $Z-P$ parameter space for different frequencies across all play conditions. It can be seen that low-frequency nodes are characterized by a high participation coefficient $(P)$, indicating that these nodes are more likely connector nodes. Changes of different universal roles corresponding to different regions in the $Z-P$ parameter space across the frequencies are presented in Figure S1D (online only). It can be seen that the number of both provincial and especially connector hubs (connector hubs exist practically only at 2.5 and $5 \mathrm{~Hz}$ ) decreases with higher frequency. The number of nonhub connectors decreases with higher frequency, whereas the number of provincial nodes correspondingly increases. This indicates that low frequencies provide the communication within and between different modules or communities.

As displayed in Figure S2 (online only), hyperbrain networks at different frequencies show different community structures. While hyperbrain networks at frequencies above $15-20 \mathrm{~Hz}$ comprise, with some exceptions, modules separating the four brains, low-frequency networks, especially in the delta $(2.5 \mathrm{~Hz})$, theta $(5 \mathrm{~Hz})$, and alpha $(10 \mathrm{~Hz})$ frequency bands, build so-called hyperbrain modules sharing electrodes from two, three, or even four brains. Furthermore, these community structures are different at the different frequencies, leading to overlapping modularity structures when different frequencies are taken into account.

\section{Changes of network architecture across time}

To investigate changes of network architecture across time, we calculated phase coupling using a moving time window of $500 \mathrm{~ms}$ width and a $50-\mathrm{ms}$ time delay. Overall, 91 time windows related to the corresponding time points were collected by this shifting procedure. We present here exemplarily a sequence from Libertango and a sequence from Comme un Tango. In the first sequence of Libertango, 
A

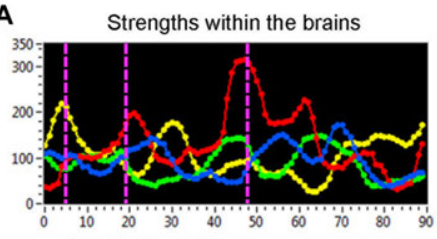

$A->B, C$, and D

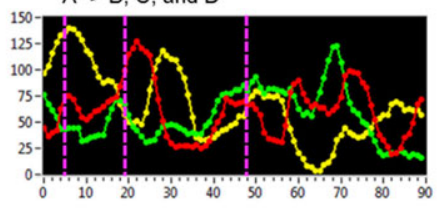

D $\rightarrow$ A, B, and C

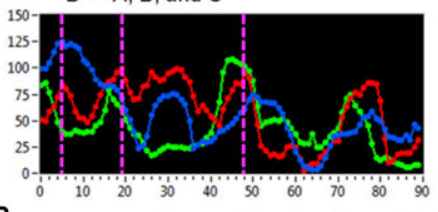

Connectivity strength and its distribution at three different time points

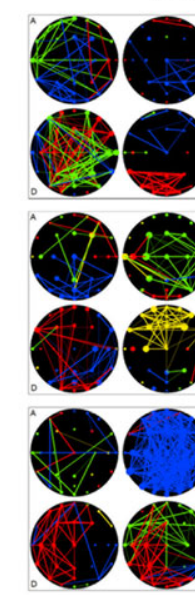

C

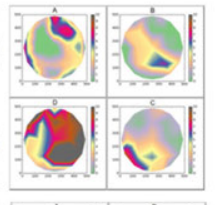

5

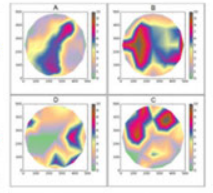

19
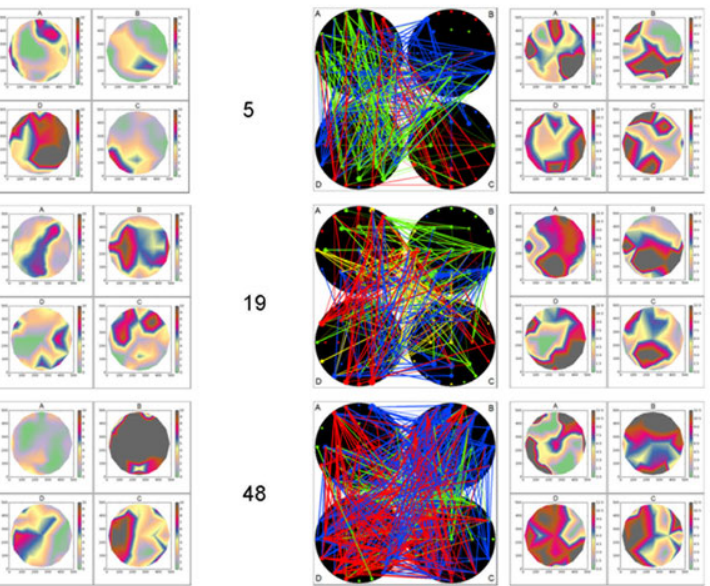

$Z-P$ parameter space at three different time points
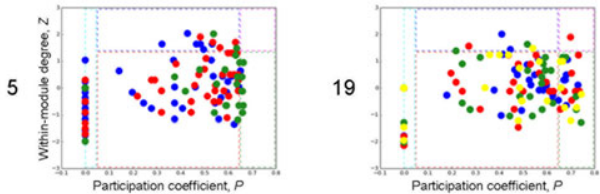

48

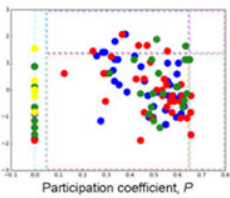

Figure 3. Temporal changes in intra- and interbrain strengths, brain connectivity and topology distribution maps, and $Z-P$ parameter space scatter plots at three different time points for the FOI $2.5 \mathrm{~Hz}$ (musical sequence 1 of Libertango). (A) Changes in intra- and interbrain strength across time or different time windows. Changes in intrabrain strength across time were estimated for the four guitarists separately. Changes in interbrain strength across time were estimated between each of the four guitarists and all others and pairwise with regard to each guitarist separately. The four guitarists are presented by different colors: guitarist A, blue; guitarist B, red; guitarist C, green; and guitarist D, yellow. In the case of pairwise presentation (second and third rows), the color indicates to which of the guitarists the coupling is directed. (B) Brain connectivity and topology distribution maps of the four guitarists with intra- and interbrain connections (left and right columns, respectively) for three different time windows: (5) the period where only guitarist D is playing, (19) the period after the first drumming by guitarist A, and (48) the period between the drumming of guitarist $\mathrm{A}$, where the musical theme begins to be repeated. These time points or windows are indicated with dotted vertical lines in (A). The size of the circle (electrode) represents the connectivity strength, and the color of the circle and links codes the corresponding module affiliation. In the strength distribution maps, high intensity of out-strength (accumulation of the big circles) is depicted by dark red or brown. (C) $Z-P$ parameter space of the hyperbrain network for the three different time windows. The color of the circle codes its module affiliation. 
guitarist $\mathrm{D}$ is playing, and guitarists $\mathrm{A}, \mathrm{B}$, and $\mathrm{C}$ are starting to play. Moreover, guitarist A is drumming on the desk of the guitar and setting the rhythm. There are two periods in which guitarist A drums two times followed by stroking over the strings. Figure 3A displays temporal changes of strength within and between the brains for each of the quartet members (top row). In addition, betweenbrain strengths are split pairwise and combined for each guitarist displayed in the four separate diagrams. It can be seen that (1) temporal changes of strengths are oscillatory in nature and (2) the pairwise dynamic is different in different guitarist pairs, although the summary dynamic presented in the top row (strength between the brains) is quite similar in the four guitarists. In Figure 3B, we show brain maps with within- and between-brain connections and corresponding topological distribution of within- and between-brain strengths at three different time points or time windows for the FOI of $2.5 \mathrm{~Hz}$. For presentation, we have chosen three different time windows: (5) the period where only guitarist $\mathrm{D}$ is playing, (19) the period after the first drumming of guitarist $\mathrm{A}$, and (48) the period between the drummings of the guitarist $A$, where the musical theme starts to be repeated. Note that color in the connectivity maps codes affinity to different modules determined by modularity analyses. Figure $3 \mathrm{C}$ displays $Z-P$ parameter space diagrams at the three time points $(5,19$, and 48$)$ presented in Figure 3B. Five different universal roles or ranges are indicated in these diagrams: ultra-peripheral nodes (cyan), provincial nonhubs (red), provincial hubs (blue), nonhub connectors (green), and hub connectors (magenta). The distribution of these roles across the four guitarist's brains is shown in Figure S3 (online only) for Libertango and Comme un Tango at the three time points or windows.

At time point 5, when only guitarist $\mathrm{D}$ is playing, the connectivity is mostly strongest in this guitarist, both within and between the brains. Modularity analysis partials out three modules, which are all hyperbrain modules distributed across the four brains. There are a relatively high number of provincial hubs distributed across all guitarists and a relatively small number of nonhub connectors that are primarily located in guitarist D. At time point 19, in the period after the first drumming by guitarist $\mathrm{A}$, as assessed by comparably high intra- and interbrain connectivity, there are four hyperbrain modules with five provincial hubs, one hub connector (guitarist D; electrode F7), and a high number of nonhub connectors (predominantly in guitarists $\mathrm{B}$ and $\mathrm{D}$ ). The presence of hyperbrain modules sharing nodes across all four brains and a relatively high number of nonhub connectors indicate that information flow in the hyperbrain network is intra- and intermodular, and guitarist $\mathrm{D}$ plays a crucial role. At time point 48 , when the first loop of the main melody theme is over and the second loop is about to start, there is a strong increase of intrabrain strength in the brain of guitarist $\mathrm{B}$, as well as a strong increase in the interbrain connectivity in all guitarists (Fig. 3A and B). There are four hyperbrain modules, of which one (yellow) consists of only five nodes, which are all ultra-peripheral nodes and thus do not play essential roles. The other three modules are distributed across four brains and are strongly interconnected. The number of nonhub connectors is strongly reduced, but there are a relatively high number of provincial hubs; this all indicates that information flow in these modules is predominantly intramodular, wherein guitarist $\mathrm{C}$ plays a crucial role.

The chosen sequence from Comme un Tango is presented in Figure 4. In this sequence, guitarists A and $\mathrm{B}$ are consonant and leading, while guitarists $\mathrm{C}$ and $\mathrm{D}$ are accompanying. Importantly, guitarist $\mathrm{C}$ plays bass and thus sets the rhythm. As evident from Figure 4A, there is a very strong coupling from guitarist $\mathrm{C}$ to guitarist $\mathrm{A}$ (blue) in the time period between 1 and 2.5 seconds (time points 20-50). During this period, guitarists A and B (as well as guitarist D) play four notes and guitarist $C$ plays two notes. In Figure 4B, we show brain maps with within- and between-brain connections and corresponding topological distribution of withinand between-brain strengths at three different time points $(28,36$, and 64$)$. The distribution of universal roles across the four guitarists' brains can be seen in Figure S3 (online only). At time point 28, all guitarists play a note and are strongly synchronized with each other. The modularity structure consists of five modules, and one of the modules (cyan) consists of only three nodes, which are all ultraperipheral nodes and thus do not play essential roles. As shown in Figure 4C and S3 (online only), there are two hub connectors (Fpl and F3 electrodes in guitarist $\mathrm{C}$ ) and a high number of nonhub connectors, which support intermodular connectivity, especially between the brains (compare intra- and 
A

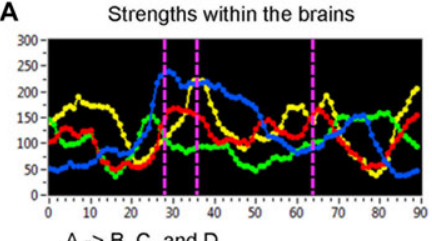
$A \rightarrow B, C$, and D
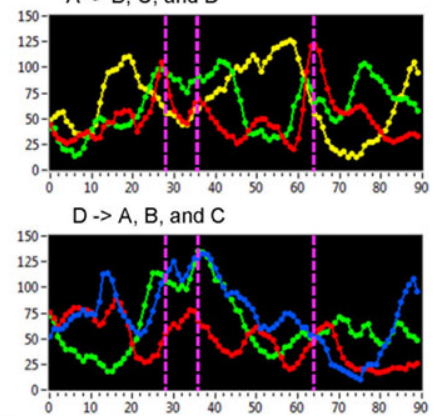

Strengths between the brains

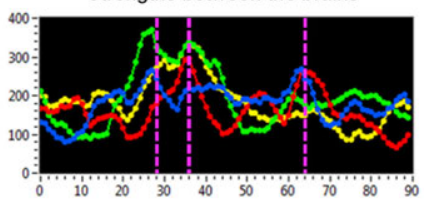

$$
B \rightarrow A, C \text {, and D }
$$

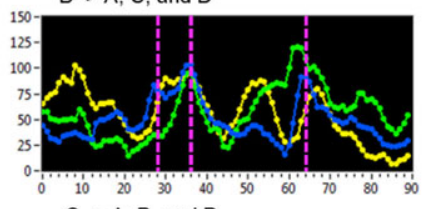

$C \rightarrow A, B$, and D

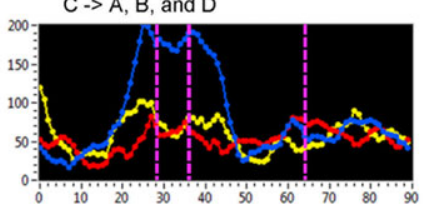

B Connectivity strength and its distribution at three different time points

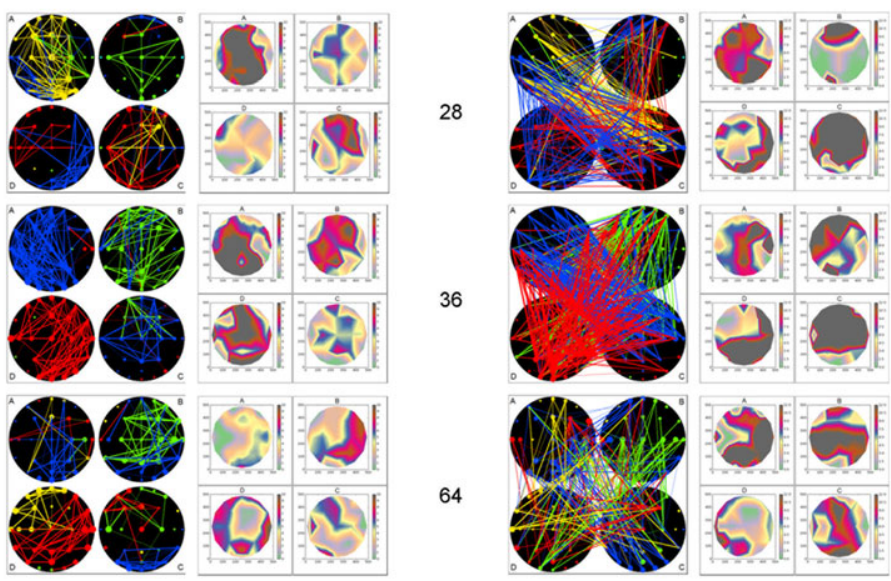

C

Z-P parameter space at three different time points

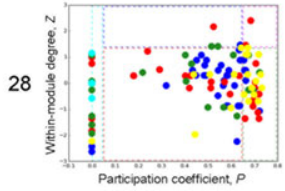

36

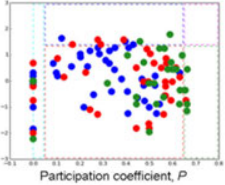

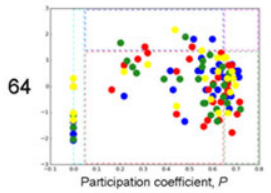

Figure 4. Temporal changes in intra- and interbrain strengths, brain connectivity and topology distribution maps, and $Z-P$ parameter space scatter plots at three different time points for the FOI $2.5 \mathrm{~Hz}$ (musical sequence 3 of Comme un Tango). (A) Changes in intra- and interbrain strength across time or different time windows. Changes in intrabrain strength across time were estimated for the four guitarists separately. Changes in interbrain strength across time were estimated between each of the four guitarists and all others and pairwise with regard to each guitarist separately. The four guitarists are presented by different colors: guitarist A, blue; guitarist B, red; guitarist C, green; and guitarist D, yellow. In the case of pairwise presentation (second and third rows), the color indicates to which of the guitarists the coupling is directed. (B) Brain connectivity and topology distribution maps of the four guitarists with intra- and interbrain connections (left and right columns, respectively) for three different time windows: (28) the period where all guitarists play a note and are strongly synchronized with each other, (36) the period where guitarists A and B (as well as guitarist D) play the next note, and (64) the period where the consonant stream of the guitarists A and B starts to decay but guitarists $\mathrm{C}$ and $\mathrm{D}$ continue with their playing (guitarist $\mathrm{D}$ even starts to play a note). These time points or windows are indicated with dotted vertical lines in (A). The size of the circle (electrode) represents the connectivity strength, and the color of the circle and links codes the corresponding module affiliation. In the strength distribution maps, high intensity of out-strength (accumulation of the big circles) is depicted by dark red or brown. (C) Z-P parameter space of the hyperbrain network for the three different time windows. The color of the circle codes its module affiliation. 
interbrain connectivity maps in Fig. 4B). At time point 36 , guitarists $A$ and $B$ (but also guitarist $D$ ) play the next note, and all four guitarists became strongly interconnected. Interestingly, the modularity structure shows only three modules distributed across the four brains. The number of nonhub connectors is reduced, indicating that information flow is predominantly intramodular (Fig. 4C). At time point 64 , the consonant streams of the guitarists $\mathrm{A}$ and $B$ start to decay, but guitarists $C$ and $D$ continue with their playing (guitarist $\mathrm{D}$ even starts to play a note). Interestingly, guitarists $\mathrm{A}$ and $\mathrm{B}$ showed the highest out-strengths (Fig. 4A and B) during this period. The modularity structure contains four hyperbrain modules, with a high number of nonhub connectors providing for high intermodular information flow.

Overall, it can be seen that the network architecture is never stable: both coupling strengths and community structures change their patterns dependent on the musical situation. Coupling strength oscillates and exhibits second-order oscillations, which can also evolve synchronously. Most importantly, most of the community structures at all three frequencies presented here (see similar presentation of strength dynamics and network architecture for theta and alpha frequency bins (i.e., 5 and $10 \mathrm{~Hz}$ ) in Figs. S4-S7, online only) are hyperbrain modules sharing electrodes from different brains. The inspection of community structures at other frequencies showed that, in contrast to previous analyses taking into account the whole segment, the hyperbrain modules are also observable at higher frequencies, indicating that high frequencies are also suitable for interbrain communication, although to a lesser extent than low frequencies.

\section{Discussion}

The primary objective of this study was to investigate the intra- and interbrain dynamics and hyperbrain architecture in a quartet of guitarists playing together. The main findings are that (1) hyperbrain networks during playing guitar in quartet are SWNs, at least for $10-50 \%$ of costs, whereby local and global efficiency increase with higher costs (lower threshold), indicating increasing segregation and integration in these networks with higher wiring costs; (2) SWN characteristics vary as a function of oscillation frequency, indicating networks with more random characteristics at low frequencies and with more regular characteristics at high frequencies; (3) in accordance with our earlier findings, ${ }^{9}$ intrabrain coupling strengths increase and interbrain coupling strengths decrease with higher frequency; (4) the hyperbrain networks consist of an overlapping modular organization changing across frequency and time; (5) the dynamics of connectivity strength and modular structures are nonstationary and contingent on musical situation or other IAC requirements, whereby coupling strength oscillates and exhibits second-order oscillations; and (6) the most important characteristic of the hyperbrain network organization is the existence of so-called hyperbrain modules sharing electrodes from two, three, or even four brains and characterized by strong connections or information flow within the modules and weak connections or information flow between the modules; such attuned modular organization of hyperbrain networks reflects intra- and interbrain flexibility and provides efficient information flow within and between the brains. These modules are sufficiently independent to guarantee functional specialization and sufficiently connected to bind multiple processes during IAC. This intramodular connectivity is sometimes supplemented by intermodular connections via connector nodes, which are characterized by enhanced connectivity to other modules. Intermodular connectivity can play a specific role during IAC, providing communication between different processes running in the interacting brains.

We presented various network metrics (CC, CPL, $E_{\text {local }}$, and, $E_{\text {global }}$, ) and SW characteristics $(\sigma$ and $\omega)$ as functions of costs among real, regular (lattice), and random networks. Real networks possess much higher global efficiency than regular networks but lower global efficiency than random networks. Thus, the hyperbrain networks of the guitarist quartet show economically plausible features (i.e., high local and global efficiency of parallel information processing on the basis of low network connection costs) at practically all frequencies investigated in the study, in line with other biological and social systems. $^{22-24}$ SW coefficients $(\sigma$ and $\omega$ ) indicate that the hyperbrain quartet network is an $\mathrm{SWN}$ at all costs (at least until the $50 \%$ cost presented here) and at all frequencies. The SW coefficient $\omega$ decreases continuously with higher frequency and lies in the positive range at low frequencies $(2.5-15 \mathrm{~Hz})$ and 
the negative range at high frequencies $(20-60 \mathrm{~Hz})$, indicating an SWN with more random characteristics at low frequencies and with more regular characteristics at high frequencies. These results are consistent with our previous study on guitar duets. ${ }^{9}$ Thus, it seems to be a general tendency of hyperbrain networks to arise during IAC, at least when playing guitar. SWNs combine high clustering and short path length between nodes, are often found in nervous systems, ${ }^{25}$ and permit high local and global information processing efficiency within and between systems. SWNs reflect two fundamental principles of brain function: segregation and integration. $^{26}$

We also investigated changes of network architecture across time using a sliding time window. It has been shown that the network architecture is never stable: both coupling strengths and community structures change their patterns over time, dependent on the musical situation. For instance, in a situation where only guitarist $\mathrm{D}$ is playing, we observed an increase in the within-brain strength of this guitarist, together with an increase in the interbrain strengths of this guitarist in either direction. In the middle of the sequence, when the second loop of the main melody theme takes place, the within-brain coupling strongly increased in guitarist $\mathrm{B}$, accompanied by a strong increase of between-brain coupling in all guitarists. Changes in within- and betweenbrain connectivity or strength are accompanied by corresponding changes in modular organization of the quartet hyperbrain network and information flow within and between the different modules integrated in a common hyperbrain network. All of these changes indicate that the hyperbrain network consisting of four different brains is a dynamic structure with nonstationary coupling dynamics and network architecture, which are highly adaptive to external situations requiring different network states. Changes in the dynamic repertoire of network states and the existence of temporally persistent network edges and motifs found in several studies on functional brain connectivity ${ }^{27-30}$ are consistent with previous studies about "EEG microstates"31-34 and metastability of brain networks. ${ }^{35,36}$ Furthermore, differences in connectivity patterns, topological distribution of the strengths, and modular organization across the four brains suggest that the brain of each guitarist plays a specific role in the hyperbrain network rather than being driven by the same shared sensory experience. Nevertheless, the influence of the latter is certainly present as well. We propose that dynamic changes of coupling strength or so-called second-order oscillations may be related to musical rhythm or other characteristics of the music and that the individual brains develop different synchronization patterns, that is, process these characteristics of the music differently.

\section{Limitations}

The present experiment has limitations and leaves ample room for questions to be addressed in future research. First, we only investigated hyperbrain network properties in one quartet. However, the main patterns of hyperbrain connectivity and network organization were replicated across different musical sequences in two different music pieces we investigated. Second, our analyses were limited to phase synchronization within single frequencies. Crossfrequency coupling analyses are likely to provide further information about functionally relevant network properties. ${ }^{20,37}$

\section{Conclusions}

Our results show that hyperbrain networks constructed on the basis of intra- and interbrain connectivity across four brains offer a new approach to investigate IAC, with more complex intertwined network structures that cannot be investigated in dual hyperbrain networks. We showed that these networks differ across frequencies and time, as well as across different musical sequences. To obtain a more complete understanding of what is happening during IAC, it is indispensable to analyze dynamic changes in synchronization and network architecture. In doing so, the present study demonstrates again that EEG hyperscanning followed by the topographical analysis of oscillatory multibrain networks is an important tool for delineating the neural mechanisms of IAC.

\section{Acknowledgments}

We thank the guitarists from the Cuarteto Apasionado for participation in the study. The authors are grateful to Bibiana Klempova, Charlotte Küpper, Anna Würtz, and Christine Wolter for technical assistance and for carrying out the experimental part of the study. We thank Daphna Raz for professional musical support in evaluation of the study 
results and also Berndt Wischnewski for software assistance.

\section{Supporting Information}

Additional supporting information may be found in the online version of this article.

Supplementary Materials. Methods.

Table S1. Music 5-s sequences in Libertango and Comme un Tango.

Table S2. Mean and standard deviation of the modularity values for real and random networks.

Table S3. ANOVA results for the coupling outstrength across the different sequences $(N=24)$.

Figure S1. Changes in modularity structure across different wiring costs and frequency bins.

Figure S2. Modular organization of hyperbrain networks at different frequency bins (the musical sequence 1 of Libertango).

Figure S3. Distribution of universal roles of $Z-P$ parameter space across the four guitarist's brains for Libertango and Comme un Tango at the three different time points.

Figure S4. Temporal changes in intra- and interbrain strengths, brain connectivity and topology distribution maps, and $Z-P$ parameter space scatter plots at three different time points for the FOI $5 \mathrm{~Hz}$ (musical sequence 1 of Libertango).

Figure S5. Temporal changes in intra- and interbrain strengths, brain connectivity and topology distribution maps, and $Z-P$ parameter space scatter plots at three different time points for the FOI $10 \mathrm{~Hz}$ (musical sequence 1 of Libertango).

Figure S6. Temporal changes in intra- and interbrain strengths, brain connectivity and topology distribution maps, and $Z-P$ parameter space scatter plots at three different time points for the FOI $5 \mathrm{~Hz}$ (musical sequence 3 of Comme un Tango).

Figure S7. Temporal changes in intra- and interbrain strengths, brain connectivity and topology distribution maps, and $Z-P$ parameter space scatter plots at three different time points for the FOI $10 \mathrm{~Hz}$ (musical sequence 3 of Comme un Tango).

\section{Competing interests}

The authors declare no competing interests.

\section{References}

1. Dumas, G., J. Nadel, R. Soussignan, et al. 2010. Interbrain synchronization during social interaction. PLoS One 5: e12166.

2. Hari, R. \& M.V. Kujala. 2009. Brain basis of human social interaction: from concepts to brain imaging. Physiol. Rev. 89: 453-479.

3. Hasson, U., A.A. Ghazanfar, B. Galantucci, et al. 2012. Brainto-brain coupling: a mechanism for creating and sharing a social world. Trends Cogn. Sci. 16: 114-121.

4. Sänger, J., U. Lindenberger \& V. Müller. 2011. Interactive brains, social minds. Commun. Integr. Biol. 4: 655-663.

5. Müller, V. \& U. Lindenberger. 2011. Cardiac and respiratory patterns synchronize between persons during choir singing. PLoS One 6: e24893.

6. Frith, U. \& C.D. Frith. 2003. Development and neurophysiology of mentalizing. Philos. Trans. R. Soc. B Biol. Sci. 358: 459-473.

7. Thompson, E. \& F.J. Varela. 2001. Radical embodiment: neural dynamics and consciousness. Trends Cogn. Sci. 5: 418425.

8. Lindenberger, U., S.-C. Li, W. Gruber, et al. 2009. Brains swinging in concert: cortical phase synchronization while playing guitar. BMC Neurosci. 10: 22.

9. Müller, V., J. Sänger \& U. Lindenberger. 2013. Intra- and inter-brain synchronization during musical improvisation on the guitar. PLoS One 8: e73852.

10. Sänger, J., V. Müller \& U. Lindenberger. 2012. Intra- and interbrain synchronization and network properties when playing guitar in duets. Front. Hum. Neurosci. 6: 312.

11. Sänger, J., V. Müller \& U. Lindenberger. 2013. Directionality in hyperbrain networks discriminates between leaders and followers in guitar duets. Front. Hum. Neurosci. 7: 234.

12. Guimerà, R. \& L.A.N. Amaral. 2005. Functional cartography of complex metabolic networks. Nature 433: 895-900.

13. Leicht, E. \& M. Newman. 2008. Community structure in directed networks. Phys. Rev. Lett. 100: 118703.

14. Newman, M. \& M. Girvan. 2004. Finding and evaluating community structure in networks. Phys. Rev. E 69: 26113.

15. Astolfi, L., J. Toppi, F. De Vico Fallani, et al. 2010. Neuroelectrical hyperscanning measures simultaneous brain activity in humans. Brain Topogr. 23: 243-256.

16. Babiloni, C., P. Buffo, F. Vecchio, et al. 2012. Brains "in concert": frontal oscillatory alpha rhythms and empathy in professional musicians. Neuroimage 60: 105-116.

17. Dikker, S., L. Wan, I. Davidesco, et al. 2017. Brain-to-brain synchrony tracks real-world dynamic group interactions in the classroom. Curr. Biol. 27: 1375-1380.

18. Chang, A., S.R. Livingstone, D.J. Bosnyak, et al. 2017. Body sway reflects leadership in joint music performance. Proc. Natl. Acad. Sci. USA 114: E4134-E4141.

19. Vigário, R.N. 1997. Extraction of ocular artefacts from EEG using independent component analysis. Electroencephalogr. Clin. Neurophysiol. 103: 395-404.

20. Müller, V. \& U. Lindenberger. 2014. Hyper-brain networks support romantic kissing in humans. PLoS One 9: e112080.

21. Bassett, D.S., D.L. Greenfield, A. Meyer-Lindenberg, et al. 2010. Efficient physical embedding of topologically complex 
information processing networks in brains and computer circuits. PLoS Comput. Biol. 6: e1000748.

22. Achard, S. \& E. Bullmore. 2007. Efficiency and cost of economical brain functional networks. PLoS Comput. Biol. 3: e17.

23. Joudaki, A., N. Salehi, M. Jalili, et al. 2012. EEG-based functional brain networks: does the network size matter? PLoS One 7: e35673.

24. Lin, C.-S., Y. Liu, W.-Y. Huang, et al. 2013. Sculpting the intrinsic modular organization of spontaneous brain activity by art. PLoS One 8: e66761.

25. Fornito, A., A. Zalesky \& E. Bullmore. 2016. Fundamentals of Brain Network Analysis. Elsevier.

26. Tononi, G. \& G.M. Edelman. 1998. Consciousness and complexity. Science 282: 1846-1851.

27. Betzel, R.F., M.A. Erickson, M. Abell, et al. 2012. Synchronization dynamics and evidence for a repertoire of network states in resting EEG. Front. Comput. Neurosci. 6: 74.

28. Damaraju, E., E.A. Allen, A. Belger, et al. 2014. Dynamic functional connectivity analysis reveals transient states of dysconnectivity in schizophrenia. Neuroimage Clin. 5: 298308.

29. De Vico Fallani, F., V. Latora, L. Astolfi, et al. 2008. Persistent patterns of interconnection in time-varying cortical networks estimated from high-resolution EEG recordings in humans during a simple motor act. J. Phys. A Math. Theor. 41: 224014 .

30. Hansen, E.C.A., D. Battaglia, A. Spiegler, et al. 2015. Functional connectivity dynamics: modeling the switching behavior of the resting state. Neuroimage 105: 525-535.

31. Allefeld, C., H. Atmanspacher \& J. Wackermann. 2009. Mental states as macrostates emerging from brain electrical dynamics. Chaos 19: 15102.

32. Lehmann, D., W.K. Strik, B. Henggeler, et al. 1998. Brain electric microstates and momentary conscious mind states as building blocks of spontaneous thinking: I. Visual imagery and abstract thoughts. Int. J. Psychophysiol. 29: 1-11.

33. Stevens, A., W. Lutzenberger, D.M. Bartels, et al. 1997. Increased duration and altered topography of EEG microstates during cognitive tasks in chronic schizophrenia. Psychiatry Res. 66: 45-57.

34. Van de Ville, D., J. Britz \& C.M. Michel. 2010. EEG microstate sequences in healthy humans at rest reveal scale-free dynamics. Proc. Natl. Acad. Sci. USA 107: 18179-18184.

35. Fingelkurts, A.A. \& A.A. Fingelkurts. 2004. Making complexity simpler: multivariability and metastability in the brain. Int. J. Neurosci. 114: 843-862.

36. Tognoli, E. \& J.A.S. Kelso. 2014. The metastable brain. Neuron 81: 35-48.

37. Jirsa, V. \& V. Müller. 2013. Cross-frequency coupling in real and virtual brain networks. Front. Comput. Neurosci. 7: 78. 